\title{
Dimensional Temperature Distribution Resulting From Propagation of A Light Beam Through an Amplifying Medium
}

\author{
M. M. El-Nicklawy, A. F. Hassan, El. M. A. Nasr, A.A. Hemida, \\ S. L. Diab \& S. M. El-Genedy \\ Helwan University, Faculty of Science, Physics Department
}

\begin{abstract}
Using the Green function theory, the time dependence of the two dimensional temperature distribution resulting from the volume absorption of a pump beam incident in radial direction and an amplified laser radiation incident in axial direction was analytically determined. The mathematical derivation accounts for the effect of self-focusing generated from the laser beam. As an example computation was carried out on Ruby rod subjected to different pump configurations and laser cross-sections.
\end{abstract}

\section{Introduction:}

After the invention of high power lasers a lot of nonlinear effects was discovered as frequency doubling, bistability, three and four wave mixing, parametric amplification etc. One of these effects is the effect of self-focusing which may affect the temperature distribution in the irradiated target and it does significantly modify the prediction of theoretical analysis that leaves out selffocusing. Different authors [1-14] have studded the temperature distribution neglecting the effect of self-focusing generated in an irradiated target. This work is devoted to study the time dependent temperature distribution in two dimensions generated from the pump process and the absorption of the amplified radiation incident in axial direction in a rod placed in the focal axis of a cylinder having an elliptical cross-section. The pump source was mounted in the other focal axis. The radiation of the laser beam was considered to initiate self-focusing during its propagation in the rod. The incident laser radiation was considered to have different radii. The pump configuration was assumed to allow for different radial profile of the inverted atoms. This capability plays an important role in:

1- The energy consumption of the pump source.

2- The life time of the pump source.

3- The pump efficiency.

4- The heat generated from the pump source. 
5- The radial amplification of the incident laser beam after being amplified.

6- The heat generated from the laser beam into the radial and the axial direction of the rod.

\section{Theory}

Assuming that a homogeneous isotropic cylindrical shaped target with circular cross-section was illuminated in axial direction with a laser beam and that the target can amplify the laser radiation. Due to this assumption the target was pumped radially with a radiation originating from a gas discharge tube located in the focal axis of a cylinder with elliptical cross-section having reflecting surface. The target was placed in the other focal axis where the pump radiation was differently focused. The laser beam, which has a maximum temporally coinciding with that of the pump pulse, was considered to have the same temporal profile but with different pulse duration. Its spatial distribution was considered to have either a Gaussian shape of different constant or increasing cross-section along the propagation direction (Gaussian beam). Moreover it is assumed that a part of the pump radiation and that of the amplified laser were absorbed during their propagation within the target which was cooled radially at the outer cylinder surface.

Considering no plasma formation at the irradiated surface, and negligible multiphoton absorption the equations governing the temperature distribution are given by:

1) The heat generation equations in cylindrical coordinates

i- Originating from the pump beam

$$
\frac{\partial^{2} T_{p}(r, t)}{\partial r^{2}}+\frac{1}{r} \frac{\partial T_{p}(r, t)}{\partial r}+\frac{1}{k} g_{p}(r, t)=\frac{1}{\alpha} \frac{\partial T_{p}(r, t)}{\partial t}
$$

ii- Originating from the laser radiation

$$
\frac{\partial^{2} T_{L}(r, z, t)}{\partial r^{2}}+\frac{1}{r} \frac{\partial T_{L}(r, z, t)}{\partial r}+\frac{\partial^{2} T_{L}(r, z, t)}{\partial z^{2}}+\frac{1}{k} g_{L}(r, z, t)=\frac{1}{\alpha} \frac{\partial T_{L}(r, z, t)}{\partial t}
$$

2) The boundary condition describing the cooling at the outer radius of the rod.

$$
-\left.k \frac{\partial T_{t}(r, z, t)}{\partial r}\right|_{r=R}=\left.h T_{t}(r, z, t)\right|_{r=R}
$$

With $\mathrm{T}_{\mathrm{t}}(\mathrm{r}, \mathrm{z}, \mathrm{t})=\mathrm{T}_{\mathrm{L}}(\mathrm{r}, \mathrm{z}, \mathrm{t})+\mathrm{T}_{\mathrm{P}}(\mathrm{r}, \mathrm{t})$ 
where

$T_{P}(r, t)$ is the temperature distribution generated from the absorbed pump radiation;

$T_{L}(r, z, t)$ is the temperature distribution generated form the absorbed part of the amplified laser beam;

$\mathrm{k}$ is the thermal conductivity of the material; $\alpha=\frac{k}{\rho C_{P}}$ is the thermal diffusivity of the material, $\rho$ is the mass density of the material; $\mathrm{C}_{\mathrm{P}}$ is the specific heat of the medium by constant pressure; $g_{p}(r, t)$ is the rate of energy generated from the absorbed part of the pump beam per unit volume; $g_{L}(r, z, t)$ is the rate of energy generated from the absorbed part of the amplified laser beam per unit volume and $\mathrm{h}$ is the heat transfer coefficient.

The temperature distribution $T_{P}(r, t)$ resulting from the pump beam was found through the determination of the Green function $G_{p}\left(r, t \mid r^{\prime}, \tau\right)$

$T_{p}(r, t)=\frac{\alpha}{k} \int_{\tau=0}^{t} d \tau \int_{R} r^{\prime} G_{p}\left(r, t \mid r^{\prime}, \tau\right) g_{p}\left(r^{\prime}, \tau\right) d r^{\prime}$

with

$G_{p}\left(r, t \mid r^{\prime}, \tau\right)=\sum_{m=1}^{\infty} e^{-\alpha \beta_{m}^{2}(t-\tau)} \frac{1}{N\left(\beta_{m}\right)} J_{o}\left(\beta_{m} r\right) J_{o}\left(\beta_{m} r^{\prime}\right)$

$\beta_{m}$ are the positive roots of the equation $\beta_{m} J_{o}^{\prime}\left(\beta_{m} R\right)+H J_{o}\left(\beta_{m} R\right)=0$

$N\left(\beta_{m}\right)=\int_{0}^{R} r J_{o}^{2}\left(\beta_{m} r\right) d r=\left[\frac{2 \beta_{m}{ }^{2}}{J_{o}{ }^{2}\left(\beta_{m} R\right) R^{2}\left(H^{2}+\beta_{m}{ }^{2}\right)}\right]^{-1}$

$g_{p}(r, t)=X \int_{v_{1}}^{v_{2}} \alpha(v) G_{p}(t) I_{o p v}(v) \frac{R_{o}+R}{R_{o}+r} e^{\alpha(v)(r-R)} d v$

and

$G_{p}(t)=\frac{(n+1)^{(n+1)}}{n^{n}} \frac{t}{\Delta t_{p}}\left(1-\frac{t}{\Delta t_{p}}\right)^{n} \quad n=3 \quad 0 \leq t \leq \Delta t_{p}$ 
with $\mathrm{H}=\mathrm{h} / \mathrm{k}, \quad J_{o}\left(\beta_{m} r\right)$ and $J_{o}^{\prime}\left(\beta_{m} R\right)$ are the Bessel functions of zero order and first order respectivly, $G_{p}(t)$ is the time profile of the pump beam, $\Delta t_{p}$ is the pulse duration of the pump beam.

The temperature distribution resulting from the laser radiation is given by

$T_{L}(r, z, t)=\frac{\alpha \cdot 2 \pi}{k} \int_{r=0}^{R} \int_{\tau=0}^{t} r^{\prime} \sum_{m=0}^{\infty} \frac{2 \beta_{m}^{2}}{R^{2}\left(H^{2}+\beta_{m}^{2}\right)} \frac{1}{J_{o}^{2}\left(\beta_{m} R\right)} \exp \left[-\alpha \beta_{m}^{2}(t-\tau)\right]$

$J_{o}\left(\beta_{m} r^{\prime}\right) J_{o}\left(\beta_{m} r\right) \int_{o}^{L} G_{L}\left(z, t \mid z^{\prime}, \tau\right) \cdot g_{L}\left(r^{\prime}, z^{\prime}, \tau\right) d r^{\prime} d z^{\prime} d \tau$

with

$G_{L}\left(z, t \mid z^{\prime}, \tau\right)=\frac{1}{2 \sqrt{\alpha} \sqrt{\pi} \sqrt{t-\tau}}\left[\exp -\left[\left(\frac{2 L-z-z^{\prime}}{2 \sqrt{\alpha}}\right)^{2} \frac{1}{t-\tau}\right]+\exp -\left[\left(\frac{z+z^{\prime}}{2 \sqrt{\alpha}}\right)^{2} \frac{1}{t-\tau}\right]+\right.$

$\exp -\left[\left(\frac{2 L-z^{\prime}+z}{2 \sqrt{\alpha}}\right)^{2} \frac{1}{t-\tau}\right]+\exp -\left[\left(\frac{z-z^{\prime}}{2 \sqrt{\alpha}}\right)^{2} \frac{1}{t-\tau}\right]+\exp -\left[\left(\frac{2 L+z-z^{\prime}}{2 \sqrt{\alpha}}\right)^{2} \frac{1}{t-\tau}\right]+$

$\exp -\left[\left(\frac{4 L-z-z^{\prime}}{2 \sqrt{\alpha}}\right)^{2} \frac{1}{t-\tau}\right]+\exp -\left[\left(\frac{z+z^{\prime}+2 L}{2 \sqrt{\alpha}}\right)^{2} \frac{1}{t-\tau}\right]+\exp -\left[\left(\frac{4 L-z^{\prime}+z}{2 \sqrt{\alpha}}\right)^{2} \frac{1}{t-\tau}\right]$

$g_{L}(r, z, t)=\int_{v} I_{o L v}(v, r, 0, t) \alpha_{h} \exp \left(-\left(\alpha_{L}(v, r, t)-\beta_{L}(v, r, t)\right) z\right) d v$

$I_{o L v}(v, r, 0, t)=\tilde{I}_{o} g_{L}\left(v, v_{o L}\right) g_{L}(r) \cdot G_{L}(t)$

and

$G_{L}(t)=\frac{(n+1)^{n+1}}{n^{n}} \frac{\left(t-\Delta t_{o}\right)}{\Delta t_{L}}\left(1-\left(t-\Delta t_{o}\right) / \Delta t_{L}\right)^{n}$

where

$I_{o L v}(v, r, 0, t)$ is the spectral intensity distribution of the laser beam incident in axial direction on the pumped laser $\operatorname{rod}$ at $\mathrm{z}=0, \alpha_{\mathrm{h}}$ is the coefficient responsible for the heating process it has according to [7] the value $201 / \mathrm{m}$, $\alpha_{\mathrm{L}}(v, \mathrm{r}, \mathrm{t})$ and $\beta_{\mathrm{L}}(v, \mathrm{r}, \mathrm{t})$ are the linear attenuation and amplification coefficient of the pumped rod in the frequency region of the incident laser radiation respectively, $\tilde{I}_{o}$ is the maximum of the incident laser intensity, 
$g_{L}\left(v, v_{o L}\right)$ is the spatial line profile of the laser beam with the central frequency $v_{o L}, G_{L}(t)$ is the temporal profile of the laser beam, $\Delta t_{o}$ is the time retardation for coinciding the maximum of the pump beam with the laser beam, $\Delta t_{L}$ is the pulse duration of the laser beam, $g_{L}(r)$ is the radial distribution of the laser radiation given for the case of Gaussian distribution when self-focusing is not considered by :

$g_{L}(r)=e^{-2 \frac{r^{2}}{w^{2}}}$

$g_{L}(r, z)$ is the radial distribution of the laser radiation given for the Gaussian distribution and Gaussian beam considering self-focusing by $g_{L}(r, z)=\frac{w_{0}^{2}}{w(z)^{2}} e^{-2 \frac{r^{2}}{w(z)^{2}}}$

where $w_{o}$ is the width of the laser beam at the irradiated surface of the rod at $\mathrm{z}=0$ and $\mathrm{w}(\mathrm{z})$ is the width at any location $\mathrm{z}$ within the rod, $w_{o}$ and $\mathrm{w}(\mathrm{z})$ in the cases of Gaussian distribution and Gaussian beam have to be determined as follows.

Self focusing is a focusing of a beam, caused by the beam itself through a nonlinear process. In this situation, the higher intensities on the beam axis, as compared to the wings of the spatial intensity distribution, cause an effectively increased refractive index for the inner part of the beam. This modified refractive index distribution then acts like a focusing lens. As the beam radius is reduced, the optical intensities become even higher, leading to further increase of the self-focusing. This mechanism can lead to very high optical intensities which can easily destroy the optical medium.

The nonlinear refractive index of a medium is given by [17]

$$
n=n_{0}+n_{2} E^{2} \quad \text { with } \quad E=\frac{1}{2}\left(\hat{E}(\omega) e^{-i \omega t}+c c\right)
$$

where $\mathrm{E}$ is the electric field amplitude of the light beam $\mathrm{n}_{2}$ is a material constant and $n_{0}$ is the refractive index when the field is very week. The dependence of the beam width on the distance covered by the beam in a medium can be obtained from Maxwell's equation. The wave equation of the electric field when 
the square of the refractive index given by equation (17) is approximated for $\mathrm{n}_{0}$ $>>\mathrm{n}_{2}$ by $n^{2} \approx n_{0}^{2}+2 n_{0} n_{2} E^{2}$ can be written as[17]:

$$
\nabla^{2} E-\frac{n^{2}}{c^{2}} \frac{\partial^{2} E}{\partial t^{2}} \approx \nabla^{2} E-\frac{1}{c^{2}}\left(n_{0}^{2}+2 n_{0} n_{2} E^{2}\right) \frac{\partial^{2} E}{\partial t^{2}}=0
$$

Equation (18) becomes in paraxial approximation after averaging over an optical period[17]

$$
\nabla_{T}^{2} \hat{E}+2 i k \frac{d \hat{E}}{d z}+\frac{k^{2} n_{2}}{n_{o}}|\hat{E}|^{2} \hat{E}=0
$$

where $\nabla_{T}^{2}$ is the transverse Laplacian

Since the dependence of the width $\mathrm{w}$ on $\mathrm{z}$ is required equation (19) has to be solved.

Assuming the solution of equation (19) to be given by

$$
\hat{E}(\vec{r})=A(\vec{r}) e^{i k s(r)}
$$

where $\mathrm{A}$ and $\mathrm{S}$ are real functions.

Equation (20) and (19) give

$$
\frac{\partial A^{2}}{\partial z}+\nabla_{T} \cdot\left(A^{2} \nabla_{T} s\right)=0
$$

and

$$
2 \frac{\partial s}{\partial z}+\left(\nabla_{T} s\right)^{2}=\frac{\nabla_{T}^{2} A}{k^{2} A}+\frac{n_{2} A^{2}}{n_{0}}
$$

Assuming $A(\vec{r})$ to be represented by a Gaussian beam given by

$$
A(\vec{r})=\frac{A_{0} w_{0}}{w(z)} e^{-r^{2} / w^{2}(z)}
$$

One gets after some mathematical manipulation the following equation[17]. 


$$
w(z)=w_{0}\left[1-\left(\frac{P_{0}}{P_{c r}}-1\right) \frac{z^{2}}{z_{0}^{2}}\right]^{1 / 2}
$$

with $\mathrm{P}_{0}$ is the incident power

$$
z_{0}=\frac{k w_{0}^{2}}{2} \text { and } P_{c r}=c \varepsilon_{0} \lambda^{2} / 8 \pi n_{2}
$$

It is worth noting that equation (24) tends to that of a Gaussian beam as $\mathrm{P}_{\mathrm{o}}$ tends to zero.

Equation (24) is valid for the case where transformation of the beam parameter resulting from its propagation through different media is not considered. Considering the transformation of $\mathrm{w}_{0}$ [17] after passing a free space of thickness $d$ and refractive index $n_{01}$ and refracted by a medium of refractive index $\mathrm{n}_{02}, \mathrm{w}(\mathrm{z})$ will be written after replacing $\mathrm{w}_{\mathrm{o}}$ by $\mathrm{w}(\mathrm{z}=0)$ given from the linear case (part I) by equation $w(z=0)=\sqrt{\frac{\lambda \tilde{L}}{2 \pi n_{01}}} \sqrt{1+\frac{\left(n_{02} \tilde{L}+2 n_{02} d\right)^{2}}{n_{02}{ }^{2} \tilde{L}^{2}}}$

where $\tilde{L}$ is the resonator length equal to the rod inside it. Thus w(z) can be written as

$$
w(z)=\sqrt{\frac{\lambda \tilde{L}}{2 \pi n_{01}}} \sqrt{1+\frac{\left(n_{02} \tilde{L}+2 n_{02} d\right)^{2}}{n_{02}{ }^{2} \tilde{L}^{2}}}\left[1-\left(\frac{p_{o}}{p_{c r}}-1\right) \frac{z^{2}}{z_{o}{ }^{2}}\right]^{1 / 2}
$$

Setting w(z) given by equation (26) in equation (16) one gets the radial distribution of the Gaussian beam case of self-focusing.

Since a Gaussian distributed laser radiation does not change its width along its propagation direction $\mathrm{z}$ as self-focusing is not considered, thus $\mathrm{w}(\mathrm{z})$ can be given by

$$
w(z)=w_{0}\left(1-\frac{p_{o}}{p_{c r}} \frac{z^{2}}{z_{0}^{2}}\right)^{1 / 2}
$$

and the radial distribution given by equation (16) will be written as $g_{L}(r, z)=\frac{w_{0}^{2}}{w(z)^{2}} e^{-2 \frac{r^{2}}{w(z)^{2}}}$

\section{Computation:}


Calculation of the temperature resulting from the absorbed laser beam $T_{L}(r, z, t)$ :

Since this work deals with medium having $\left(\beta_{L}-\alpha_{L}\right) \neq 0$, the medium was subdivided into equal spaced intervals $\Delta z=L / \mathrm{m}$. The input power of each interval was assumed to be

$P_{o} e^{\left(\beta_{L}-\alpha_{L}\right) N \Delta z}$

where $\mathrm{N}$ takes values from $\mathrm{N}=0$ to $\mathrm{m}$. $\mathrm{w}_{\mathrm{o}}$ of the radial distribution given by equation (16) was considered at the beginning of each interval to be that calculated from $\mathrm{w}(\mathrm{z})$ at the end of the previous interval. The power within the interval was considered to be constant.

To get $T_{L}(r, z, t)$ equation (10) was calculated after substituting for $G_{L}\left(z, t \mid z^{\prime}, \tau\right), g_{L}\left(r^{\prime}, z^{\prime}, \tau\right)$ and $G_{L}(t)$ from equations (11),(12) and (14) respectively. For the case of Gaussian beam the radial intensity distribution of the laser radiation was considered to be given by equation (16), after replacing $\mathrm{w}_{\mathrm{o}}$ and $\mathrm{w}(\mathrm{z})$ from equations (25) and (26) respectively. For the case of Gaussian distributed laser radiation the radial distribution is given by equation (16) but with $\mathrm{w}_{0}=$ constant and $\mathrm{w}(\mathrm{z})$ given by equation (27).

The computation was carried out considering $\mathrm{w}_{\mathrm{o}}=10^{-4} \mathrm{~m}$ for the Gaussian distribution and $\mathrm{w}_{0}=1.77 \times 10^{-4} \mathrm{~m}$ at $\mathrm{z}=0$ for the Gaussian beam and a maximum monochromatic laser intensity $\tilde{I}_{o}$ given by $\tilde{I}_{o}=10^{11} \mathrm{~W} / \mathrm{m}^{2}$ at $\mathrm{r}=0$ and $t=\Delta t_{p} / 4$.

The locations and the times at which the temperature was calculated are the multiple of the following $\Delta z, \Delta r_{i}$ and $\Delta t_{i}$ values

$\Delta z$ is considered to be given by

$\Delta z=L / 10$

$\Delta r_{i}$ values are given according to the following table

The radius of the rod $\mathrm{R}$ was according to the width of the laser beam and $\mathrm{R}_{\mathrm{o}}$ values subdivided into three zones $\Delta r_{i}$

$$
\begin{array}{rlrl}
\Delta r_{1} & =R_{o} / 10 & 0 \leq r \leq R_{o} & \\
\Delta r_{2} & =\left(w_{o}-R_{o}\right) / 10 & R_{o} \prec r \leq w_{o} \text { or } w_{o} \prec r \leq R_{o} \\
\Delta r_{3} & =\left(R-w_{o}\right) / 10 & w_{o} & \prec r \leq R
\end{array}
$$

The time was sliced into four different intervals $\Delta t_{i}$. 
$\Delta t_{1}=$ the time before initiating the laser pulse was divided by 5

$$
t=\Delta t_{1} \times n_{1} \quad n_{1}=0 \ldots \ldots 5
$$

$\Delta t_{2}=$ the time during the laser pulse duration was divided by 16

$$
t=\Delta t_{1} \times 5+\Delta t_{L} / 16 \times n_{2} \quad n_{2}=1 \ldots \ldots .16
$$

$\Delta t_{3}=$ the time calculated from the equation (the pump pulse duration (the time before initiating the laser pulse + pulse duration )) was divided by 5

$$
t=\left(\Delta t_{p}-\left(\Delta t_{1} \times 5+\Delta t_{L}\right)\right) / 5 \times n_{3}+\left(\Delta t_{1} \times 5+\Delta t_{L}\right) \quad n_{3}=\quad 1 \ldots . .5
$$

the time after switching off the pumping process was given by $\Delta t_{4}=2 \mathrm{X}$ pulse duration of the pump beam

$$
t=\Delta t_{p}+n_{4} \times \Delta t_{4} \quad n_{4}=1 \ldots \ldots .7
$$

\begin{tabular}{|c|c|c|c|c|c|c|c|c|c|c|}
\hline Symbol & $\begin{array}{l}p^{\prime} \\
\frac{\mathrm{kg}}{m^{3}}\end{array}$ & $\begin{array}{c}\mathrm{C}_{\mathrm{p}} \\
\frac{\mathrm{w} \cdot \mathrm{sec}}{\mathrm{kg} \cdot{ }^{o} \mathrm{k}}\end{array}$ & $\frac{\begin{array}{c}\mathbf{N}_{\mathbf{o}} \\
\text { ions }\end{array}}{m^{3}}$ & $\begin{array}{c}U_{01} \\
\mathrm{~Hz}\end{array}$ & $\begin{array}{c}U_{21} \\
\mathrm{~Hz}\end{array}$ & $\begin{array}{c}U_{22} \\
\mathrm{~Hz}\end{array}$ & $\begin{array}{c}U_{31} \\
\mathrm{~Hz}\end{array}$ & $\begin{array}{c}U_{32} \\
\mathrm{~Hz}\end{array}$ & $\begin{array}{c}F_{\perp}^{21} \\
1 / \mathrm{m}\end{array}$ & \\
\hline Value & 4300 & 43 & $1.58 \mathrm{E} 25$ & $1.5 \mathrm{E} 15$ & 7.3E14 & 7.1E14 & $5.4 \mathrm{E} 14$ & 5.53E14 & 320 & \\
\hline Symbol & $F_{\perp}^{31}$ & $F_{\square}^{32}$ & $F_{\square}^{01}$ & $F_{\square}^{22}$ & $\Delta v_{o}$ & $\begin{array}{l}k \\
w\end{array}$ & $\begin{array}{c}\mathrm{h} \\
\mathrm{W}\end{array}$ & $p_{c r}$ & $\mathbf{n}_{\mathbf{0}}$ & $\begin{array}{r}\mathbf{n}_{2} \\
m^{2} \\
\end{array}$ \\
\hline Unit & $1 / \mathrm{m}$ & $1 / \mathrm{m}$ & $1 / \mathrm{m}$ & & $\mathrm{Hz}$ & $m^{o} K$ & $m^{o} K$ & $w$ & & $V^{2}$ \\
\hline Value & 155 & 285 & 360 & 280 & $0.3298 \mathrm{E} 12$ & 42 & 10000 & 5000 & 1.759 & 2E-14 \\
\hline
\end{tabular}

Since the integration was carried out over $z^{\prime}, r^{\prime}$ and $\tau, \Delta z, \Delta r_{i}$ and $\Delta t_{i}$ were subdivided into 6,6,5 equal intervals respectively.

Table (1): physical and thermal parameter of Ruby:

$F_{\square \perp}{ }^{i j}$ is the absorption coefficient parallel and perpendicular to the $\mathrm{C}$-axis

of the crystal

$v_{i j}$ are the corresponding central frequency to $F^{i j}$

$p_{c r}$ is the critical power

$\mathrm{N}_{\mathrm{o}}$ is the $\mathrm{Cr}^{3+}$ concentration

$\mathrm{n}_{0}$ is the average refractive index of $\square$ and $\perp$ radiation at $\lambda=6943 \AA$

$\mathrm{n}_{2}$ is the coefficient of the part of the refractive index depending on square of the electric field strength.

\section{Results and Discussion:}


Figure (1) represents the radial distribution of $\left(\beta_{\mathrm{L}}-\alpha_{\mathrm{L}}\right)$ calculated at $t=\Delta t_{p} / 4$ and $\mathrm{R}_{\mathrm{o}}$ as a parameter. The observation of the curves shows that, for $\mathrm{R}_{\mathrm{o}}=10^{-4} \mathrm{~m}$ and $5 \times 10^{-4} \mathrm{~m}\left(\beta_{L}-\alpha_{L}\right)$ in the vicinity of $\mathrm{r}=0$ has a relative minimum shifted towards smaller $r$ values as $R_{o}$ increases, and that the minimum of the former $R_{0}$ value is more pronounced than that of the latter one. The curves calculated for $\mathrm{R}_{0}=10^{-3} \mathrm{~m}$ and $\infty \mathrm{m}$ grow monotonically and exhibit specially for the case $\mathrm{R}_{0}=\infty \mathrm{m}$ a saturated behavior at $\mathrm{r}=\mathrm{R}$. This behavior can be explained in view of the fact that the behavior $\left(\beta_{L}-\alpha_{L}\right)$ is dictated from the intensity distribution of the pump beam given by $\frac{R_{0}+R}{R_{0}+r} e^{\alpha(r-R)}$ which exhibits a minimum at $r=\frac{1}{\alpha}-R_{0}$. Since the value of $\alpha$ is about $10^{-3} \mathrm{~m}^{-1}$ it follows that by increasing $R_{0}$ values the position of the minimum will shift towards smaller $r$ values. As $R_{0}$ is given values greater than or equal to $1 / \alpha$ the position of the minimum will shift to such small or negative $r$ values where the relative minimum disappears. Since $\left(\beta_{L}-\alpha_{L}\right)$ at $\mathrm{r}=0$ must be $46.05 \mathrm{~m}^{-1}$, this is because an amplification of 100 at $\mathrm{z}=\mathrm{L}$ is set as a condition, and $\left(\beta_{L}-\alpha_{L}\right)$ depends on the pump energy density elsewhere, thus a minimum laying to the right of $r=0$ can have positive or negative values. In case of strong focusing the intensity of the pump radiation drops strongly with $\mathrm{r}$ leading to negative $\left(\beta_{L}-\alpha_{L}\right)$ values. The saturation at $\mathrm{r}=\mathrm{R}$ for $\mathrm{R}_{0}=\infty \mathrm{m}$ results from the behavior of $\left(\beta_{L}-\alpha_{L}\right)$ which is generally according to part $\mathrm{I}$ [18] given by $\left(\beta_{l}-\alpha_{l}\right)=c \frac{c_{1} \frac{R_{0}+R}{R_{0}+r} e^{\alpha(r-R)}-1}{c_{1} \frac{R_{0}+R}{R_{0}+r} e^{\alpha(r-R)}+1}$.

Figure (2) represents the axial distribution of the laser beam width calculated for $\mathrm{w}=10^{-4} \mathrm{~m}$ of the incident laser radiation at $t=\Delta t_{p} / 4, \mathrm{r}=0$. Since the amplification at $r=0$ is independent of $R_{0}$, therefore all the curves of the different $R_{0}$ values will coincide on each other. The figure shows that the width exhibits a minimum. The appearance of minimum is due to the dependence of $\mathrm{P}_{\mathrm{o}}$ on $\mathrm{z}$ as given by the equation $w(\mathrm{z})=w_{o} \mid\left[1-\left.\frac{P_{o}(z)}{P_{c r}} \frac{z^{2}}{z_{o}{ }^{2}}\right|^{1 / 2}\right.$. where $P_{o}(z)$ is given by equation (28). 

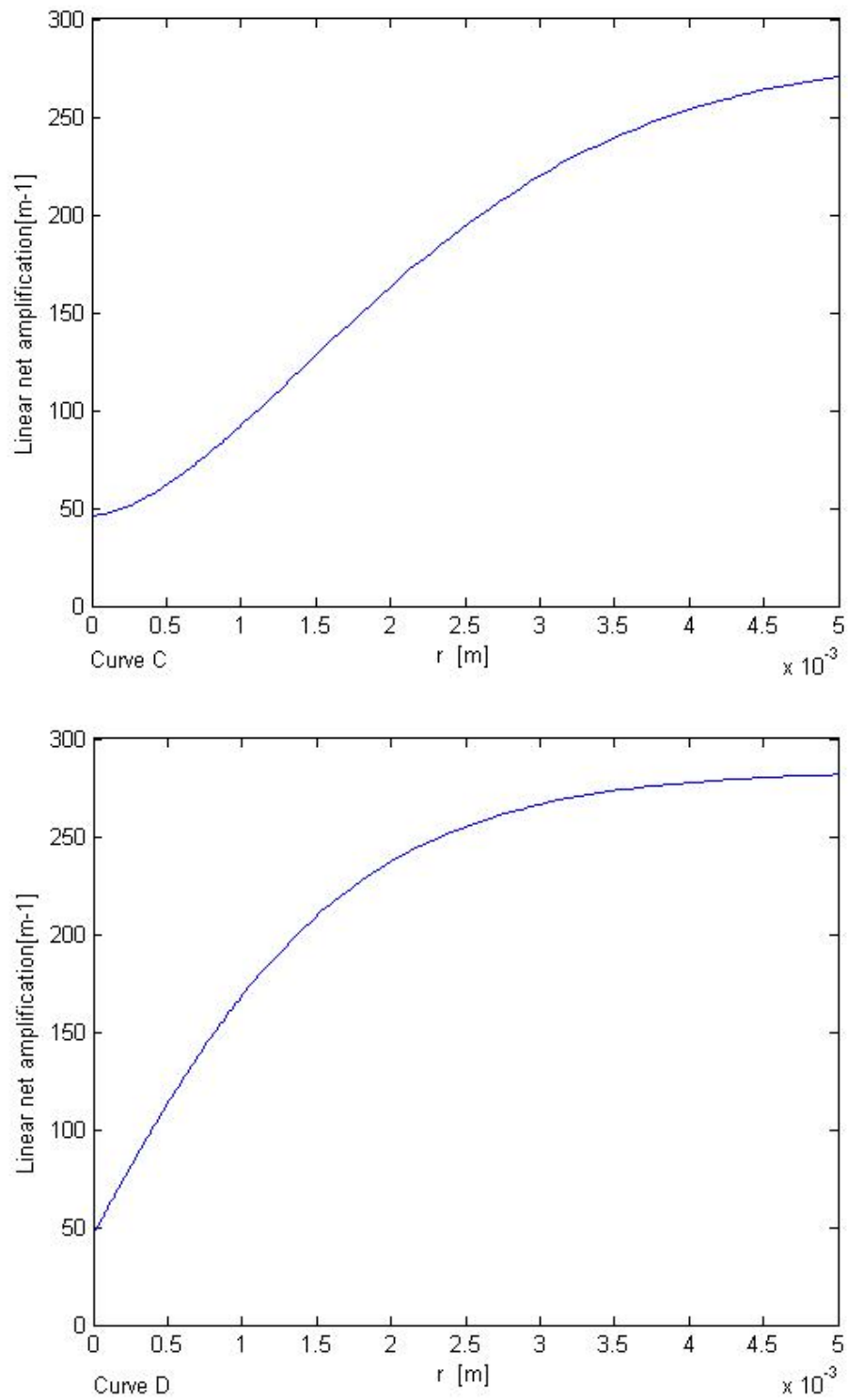

Fig. (1): The radial distribution of the net amplification coefficient of the laser radiation $\left(\beta_{\mathrm{L}}-\alpha_{\mathrm{L}}\right)$ calculated at $\mathrm{t}=\Delta \mathrm{t}_{\mathrm{p}} / 4$ with $\mathrm{R}_{0}$ as a parameter. $\mathrm{R}_{0}=10^{-4} \mathrm{~m}$ curve A $\quad \mathrm{R}_{0}=5 \times 10^{-4} \mathrm{~m}$ curve $\mathrm{B} \quad \mathrm{R}_{0}=10^{-3} \mathrm{~m}$ curve $\mathrm{C} \quad \mathrm{R}_{0}=\infty \mathrm{m}$ curve $\mathrm{D}$ 


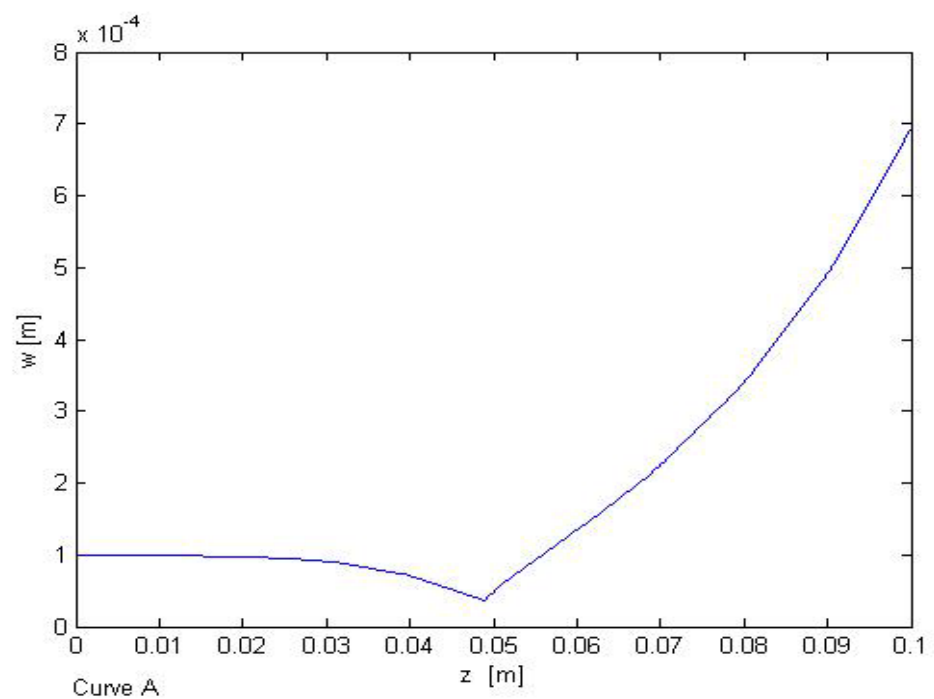

Fig. (2): The variation of the width (w) of a laser beam having a Gaussian spatial intensity distribution calculated as a function of $\mathrm{z}$. for $\mathrm{w}_{\mathrm{o}}=10^{-4} \mathrm{~m}$.

\section{Case of Gaussian distribution $\mathrm{w}=10^{-4} \mathrm{~m}$ :}

The temporal temperature distribution calculated at $\mathrm{z}=0, \mathrm{r}=0$ and $\mathrm{R}_{0}$ as a parameter, shows that the temperature behaves in relative distribution and absolute value as the corresponding ones of the linear case (part I). This behavior is due to the fact that at $\mathrm{z}=0, \mathrm{r}=0$ non of the nonlinear effects has taken place and the effect of locations other than zero is negligible small.

Figure (3) represents the temperature versus time calculated at $\mathrm{z}=\mathrm{L}, \mathrm{r}=0$ and $\mathrm{R}_{0}$ as a parameter. The curves show that the maximum of the temperature is not at $t=\Delta t_{p} / 4$ but at greater $t$ value. This behavior is for all $\mathrm{R}_{0}$ values due to the fact that before reaching the temperature its maximum value the accumulation of heat overcompensates the losses. At the maximum temperature the losses are equal to the heat generated. For times greater than the time of maximum temperature the losses are greater than the generated heat. The small slop of the time profile after switching off the laser radiation seen for $\mathrm{R}_{0}=10^{-3} \mathrm{~m}$ is due to the heat transfer from the maxima surrounding $r=0$ see Fig. (5) which leads to increase the minimum around $\mathrm{r}=0$ and decrease the heat transfer. The appearance of a minimum followed by a maximum for $\mathrm{R}_{0}=\infty \mathrm{m}$ is due to the heat transfer from $\mathrm{r}=0$ into its surrounding which as seen from Fig. (5) occurs firstly and leads to the minimum temperature. As the time increases the heat conduction from the maximum located at greater $r$-value will increase the temperature at $r=0$. This behavior lasts till the heat transfer into the region around $r=0$ is equal to the heat transfer out of this region where a maximum of the temperature occurs. After attaining the 
temperature its maximum value, it begins monotonically to decrease with small slop as $t$ increase. That the maximum of the temperature increases with increasing $\mathrm{R}_{0}$ values is due to the increased width of the laser radiation at $\mathrm{z}=\mathrm{L}$ which, as seen from Fig. (5), leads to increase the interval of the radial distribution of $\left(\beta_{L}-\alpha_{L}\right)$ contributing to the heating effect. The maximum of the temperature in this case is for $R_{0}=10^{-4} \mathrm{~m}$ and $R_{0}=5 \times 10^{-4} \mathrm{~m}$ smaller than the corresponding ones in the linear case (part I). This behavior is, for both cases, due to the increase of $\mathrm{w}(\mathrm{L})$ value which leads to a smaller intensity and it is for $R_{0}=10^{-4} \mathrm{~m}$ due to the negative behavior of the radial distribution of $\left(\beta_{L}-\alpha_{L}\right)$. For $\mathrm{R}_{0}=5 \times 10^{-4} \mathrm{~m}$ it is due to the decrease of $\left(\beta_{L}-\alpha_{L}\right)$ with increasing $\mathrm{r}$ values. For the other $\mathrm{R}_{\mathrm{o}}$ values the temperature is higher than the linear case. This is because the increase of $\mathrm{w}(\mathrm{L})$ value will cover areas having greater $\left(\beta_{L}-\alpha_{L}\right)$ values that overcompensate the decrease of the intensity.
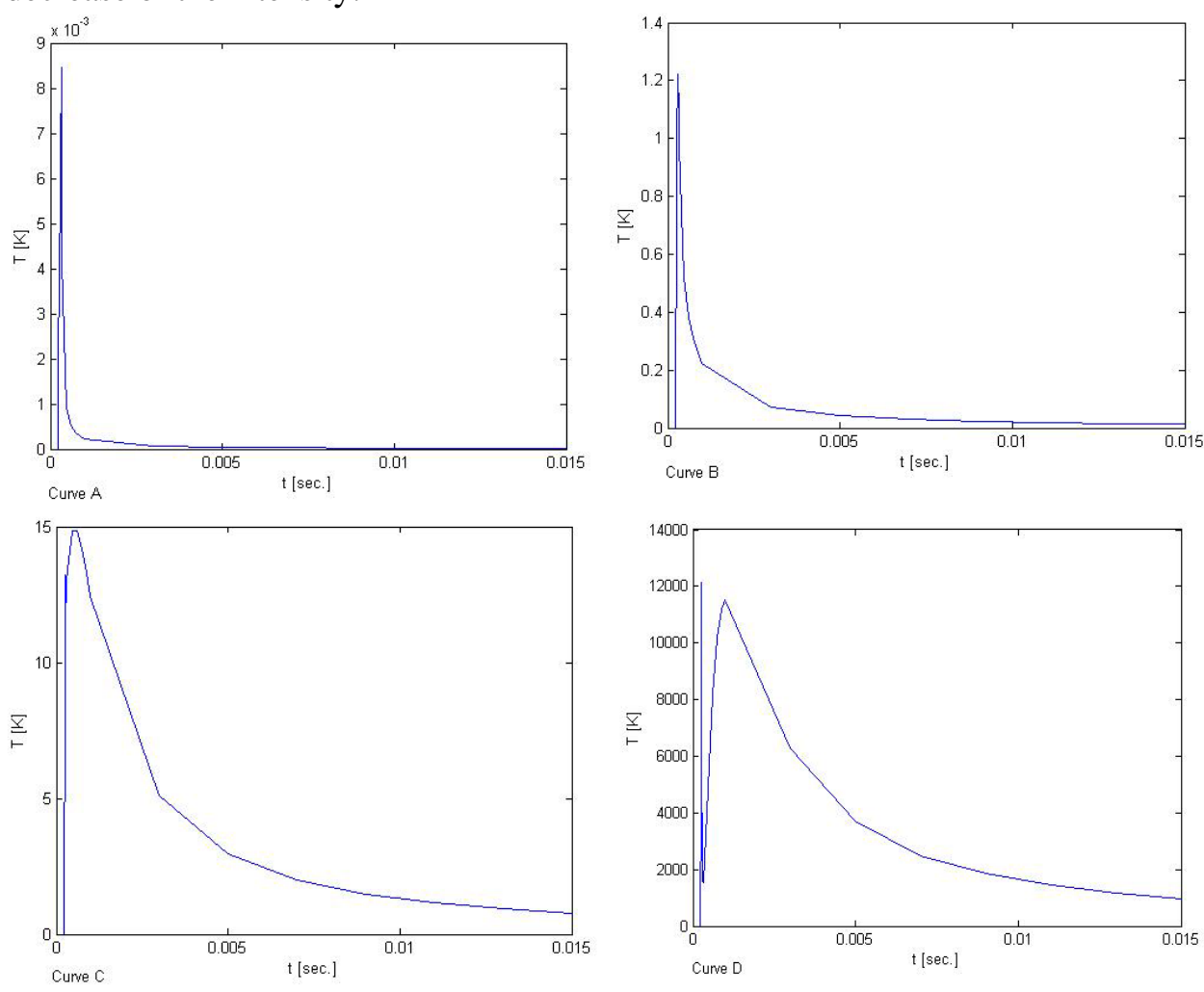

Fig. (3): The temporal temperature distribution induced by a laser beam having a Gaussian spatial intensity distribution of width $\mathrm{w}_{\mathrm{o}}=10^{-4} \mathrm{~m}$ and pulse duration $\Delta \mathrm{t}_{\mathrm{L}}=10^{-4}$ sec calculated considering self-focusing and amplified power at $\mathrm{z}=$ $\mathrm{L}, \mathrm{r}=0$ and $\mathrm{R}_{\mathrm{o}}$ as a parameter.

$\mathrm{R}_{\mathrm{o}}=10^{-4} \mathrm{~m} \quad$ curve $\mathrm{A} \quad \mathrm{R}_{\mathrm{o}}=5 \times 10^{-4} \mathrm{~m}$ curve $\mathrm{B} \quad \mathrm{R}_{\mathrm{o}}=10^{-3} \mathrm{~m} \quad$ curve $\mathrm{C}$

$\mathrm{R}_{\mathrm{o}}=\infty \mathrm{m} \quad$ curve $\mathrm{D}$ 
Figure (4) represents the temperature distribution along the $\mathrm{z}$ axis calculated at $t=\Delta t_{p} / 4, \mathrm{r}=0$ and $\mathrm{R}_{0}$ as a parameter. The case of $\mathrm{R}_{0}=10^{-4} \mathrm{~m}$ begins with higher temperature due to the great intensity. With increasing $\mathrm{z}$ the temperature decreases. This behavior is due to the negative value of $\left(\beta_{L}-\alpha_{L}\right)$ in the wings of the laser radiation which is greater than the increase of the intensity due to the reduced value of $\mathrm{w}(\mathrm{z})$ and the magnification within small $r$ values. As $\mathrm{w}(\mathrm{z})$ becomes closer to the minimum the intensity increases and the temperature becomes maximum. The further increase of $\mathrm{z}$ leads to smaller intensity (greater $\mathrm{w}(\mathrm{z})$ ) and truncation of the laser radiation due to the negative $\left(\beta_{L}-\alpha_{L}\right)$ values which can not be compensated from the increased amplification $e^{\left(\left(\beta_{L}-\alpha_{L}\right) z\right)}$ in the small remaining width of the laser radiation and the temperature decreases. For $\mathrm{R}_{0}=5 \times 10^{-4} \mathrm{~m}\left(\beta_{L}-\alpha_{L}\right)$ decreases slowly with increasing $r$ value but still remains positive. This behavior lasts up to $r=5 \times 10^{-}$ ${ }^{4} \mathrm{~m}$ after which it begins to increase. Since $\mathrm{w}(\mathrm{z})$ decreases with increasing $\mathrm{z}$ value the temperature increases monotonically. As w(z) takes great value such that the wings of the laser radiation will be amplified and the amplification compensates the decrease of the intensity and other losses, such as cooling and heat conduction, the curve attains a maximum. Further increase of $\mathrm{z}$ leads to a greater decrease of the intensity than the increase through the amplification and the temperature decreases with increasing $\mathrm{z}$ value. For $\mathrm{R}_{0}=10^{-3} \mathrm{~m}$ a monotone increase of the temperature is observed. This is due to the increased intensity before the minimum width is achieved which overcompensates the reduced radial amplification of $\left(\beta_{L}-\alpha_{L}\right)$ and the strong increase of $\mathrm{w}(\mathrm{z})$ after the minimum which covers greater radial interval from $\left(\beta_{L}-\alpha_{L}\right)$ and thus leading to greater amplification than the attenuated intensity. The same behavior is also observed for $\mathrm{R}_{0}=\infty \mathrm{m}$. Due to the increased value of $\left(\beta_{L}-\alpha_{L}\right)$ in the wings of the laser radiation as $R_{0}$ increases the maximum temperature increases with increasing $\mathrm{R}_{\mathrm{o}}$ values. Due to the greater width of the laser radiation at $\mathrm{z}=\mathrm{L}$ than the linear case and the behavior of $\left(\beta_{L}-\alpha_{L}\right)$ for $\mathrm{R}_{0}>10^{-3} \mathrm{~m}$ the temperature in this case is greater than that in the linear case (part I)

Figure (5) represents the radial temperature distribution calculated at $t=\Delta t_{p} / 4, \mathrm{z}=\mathrm{L}$ and $\mathrm{R}_{\mathrm{o}}$ as a parameter. From the figure it is found that the temperature at $r=0$ increases with increasing $R_{0}$ values. This is because of the accumulation effect of the heat over the time which is greatest for great $R_{0}$ values (higher temperature of the surrounding parts around $r=0$ ) and the smaller heat conduction in the surrounding. For $\mathrm{R}_{\mathrm{o}} \geq 10^{-3} \mathrm{~m}$ there are two maxima symmetric to the temperature at $r=0$. This is due to the increased width of the 
laser radiation which allows, due to $\left(\beta_{L}-\alpha_{L}\right)$ behavior, the wings of the laser radiation to be amplified. Since in the linear case, part[I], the value of the laser beam width is constant along the $\mathrm{z}$ axis no dip was observed.
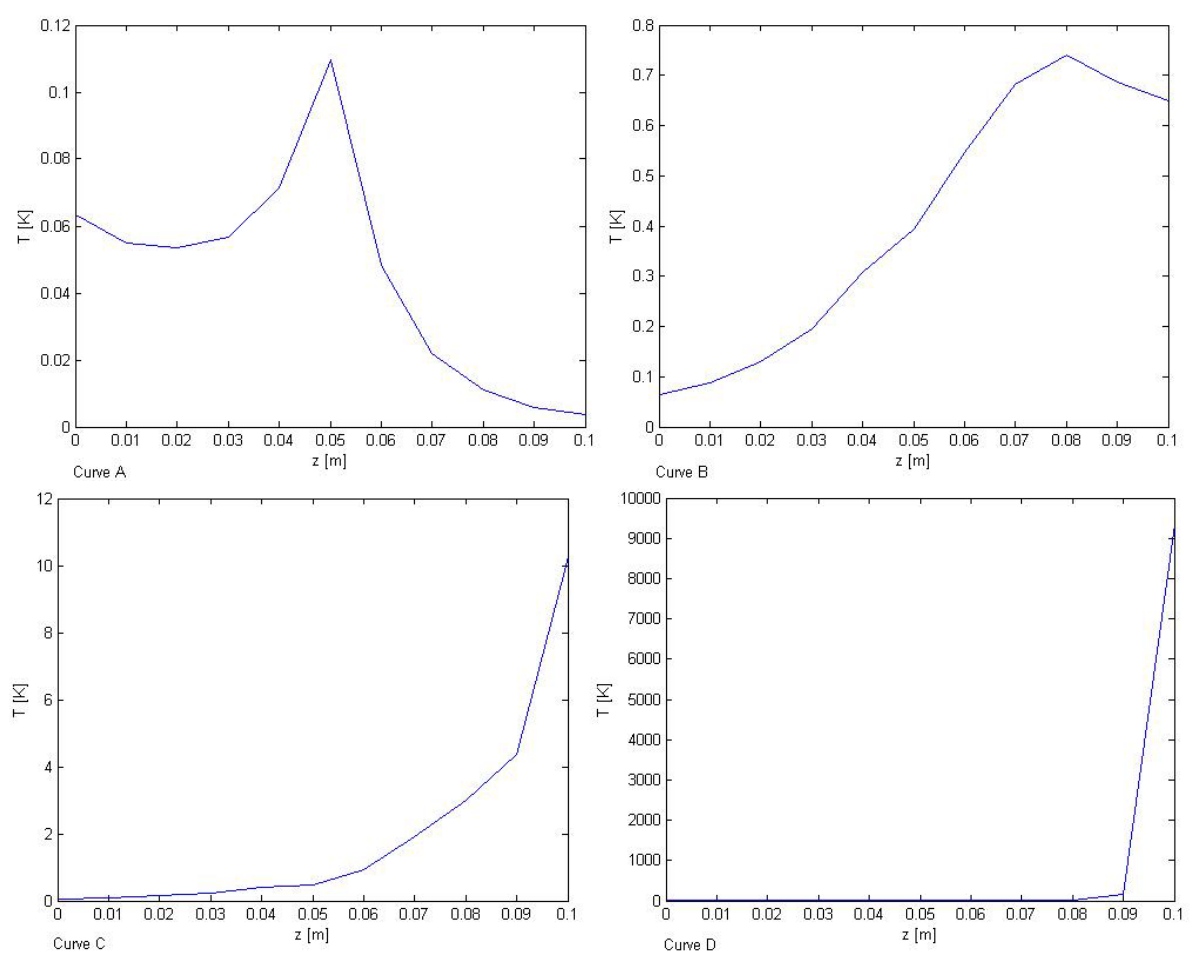

Fig. (4): The temperature distribution as a function of $\mathrm{z}$ induced by a laser beam having a Gaussian spatial intensity distribution of width $\mathrm{w}_{\mathrm{o}}=10^{-4} \mathrm{~m}$ and pulse duration $\Delta \mathrm{t}_{\mathrm{L}}=10^{-4}$ sec calculated considering self-focusing amplified power at $\mathrm{t}$ $=\Delta \mathrm{t}_{\mathrm{p}} / 4, \mathrm{r}=0$ and $\mathrm{R}_{\mathrm{o}}$ as a parameter.

$\mathrm{R}_{0}=10^{-4} \mathrm{~m} \quad$ curve $A \quad \mathrm{R}_{0}=5 \times 10^{-4} \mathrm{~m} \quad$ curve $\mathrm{B} \quad \mathrm{R}_{0}=10^{-3} \mathrm{~m} \quad$ curve $\mathrm{C}$

$\mathrm{R}_{0}=\infty \mathrm{m}$ curve $\mathrm{D}$ 

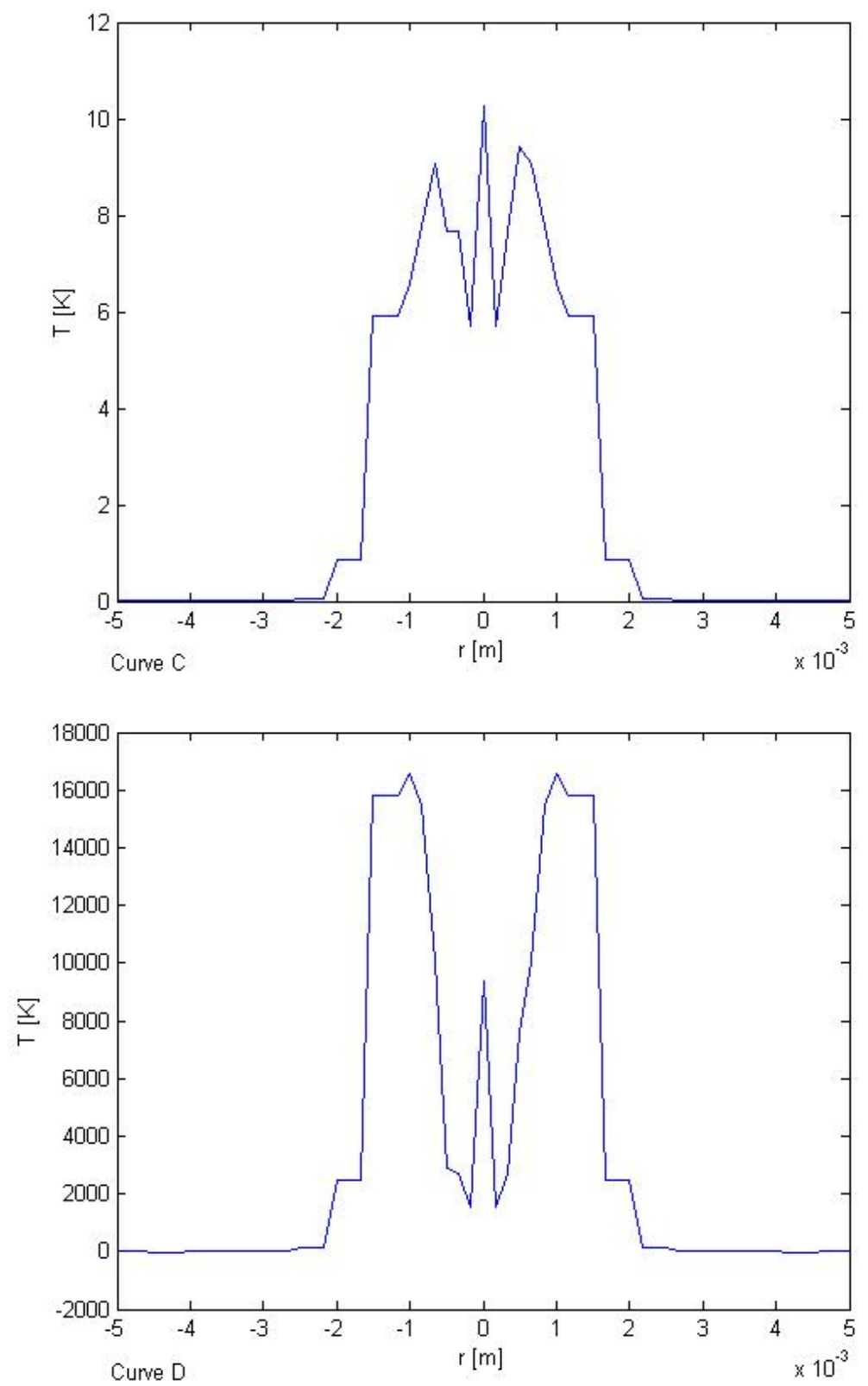

Fig. (5): The radial temperature distribution induced by a laser beam having a Gaussian spatial intensity distribution of width $\mathrm{w}_{\mathrm{o}}=10^{-4} \mathrm{~m}$ and pulse duration $\Delta \mathrm{t}_{\mathrm{L}}=10^{-4}$ sec calculated considering self-focusing amplified power at $\mathrm{t}=\Delta \mathrm{t}_{\mathrm{p}} / 4, \mathrm{z}=$ $\mathrm{L}=0.1 \mathrm{~m}$ and $\mathrm{R}_{0}$ as a parameter.

$\mathrm{R}_{\mathrm{o}}=10^{-4} \mathrm{~m} \quad$ curve $\mathrm{A} \quad \mathrm{R}_{0}=5 \times 10^{-4} \mathrm{~m} \quad$ curve $\mathrm{B} \quad \mathrm{R}_{0}=10^{-3} \mathrm{~m} \quad$ curve $\mathrm{C}$ $\mathrm{R}_{\mathrm{o}}=\infty \mathrm{m} \quad$ curve $\mathrm{D}$ 


\section{Case of Gaussian Beam:}

Figure (6) represents the axial distribution of the laser beam width $\mathrm{w}(\mathrm{z})$ given by equation (24) calculated at $t=\Delta t_{p} / 4, \mathrm{r}=0$. Due to the reasons given in Fig. (2) the curves of different $R_{o}$ values coincide on each other. This is because the amplification is a function of the radius of the pumped rod and not a function of the type of the laser radiation so far saturation effect is not considered. That the minimum width occurs at greater $\mathrm{z}$ value than the case of $\mathrm{w}_{\mathrm{o}}=10^{-4} \mathrm{~m}$ is due to the greater $\mathrm{w}_{\mathrm{o}}$ value which is equal $1.77 \times 10^{-4} \mathrm{~m}$ at $\mathrm{z}=0$ and the increasing $\mathrm{w}(\mathrm{z})$ with increasing $\mathrm{z}$ value when self-focusing is not considered. Thus due to both effects greater power is needed to get minimum width and this is achieved through the amplification of the laser radiation at greater $\mathrm{z}$ value than in case of $\mathrm{w}_{0}=10^{-4} \mathrm{~m}$.

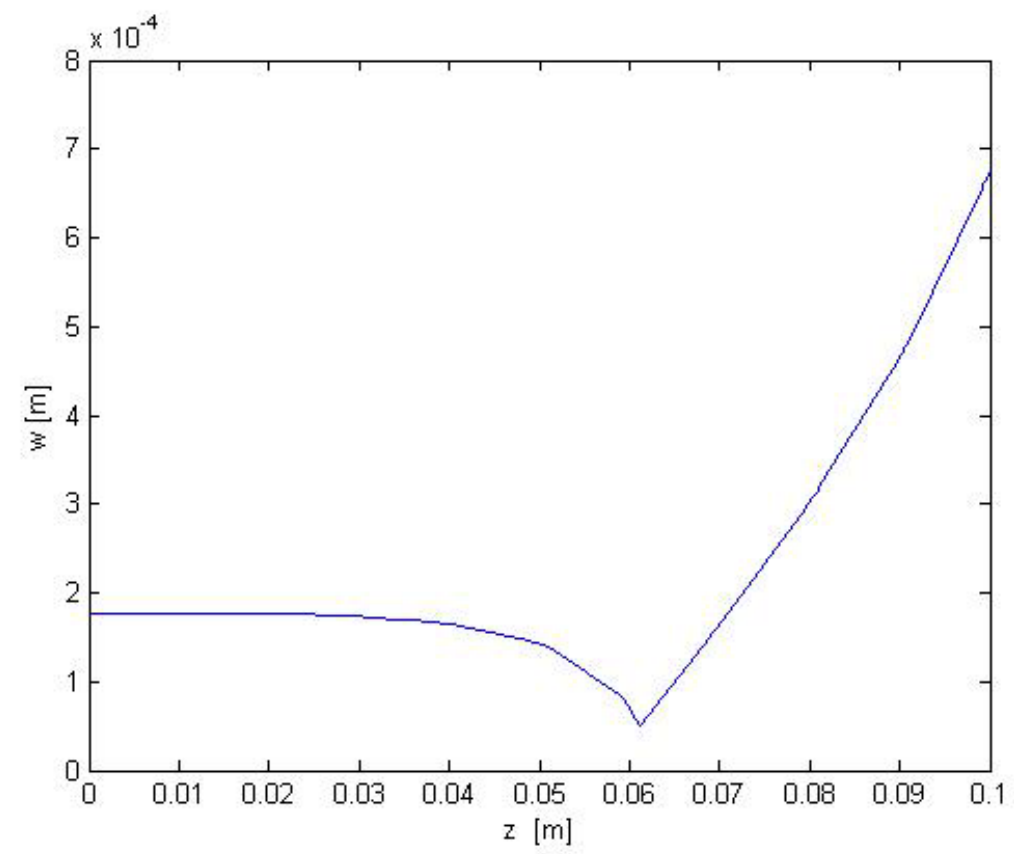

Fig. (6): The variation of the width $w$ of a laser having a Gaussian beam intensity distribution as a function of $\mathrm{z}$ calculated considering self-focusing and amplified power.

The temporal temperature distribution calculated at $\mathrm{z}=0, \mathrm{r}=0$ and $\mathrm{R}_{\mathrm{o}}$ as a parameter, shows that the temperature behaves in relative distribution and absolute value as the corresponding ones of the linear case (Part I). 
Figure (7) represents the temporal temperature distribution calculated at $\mathrm{z}=\mathrm{L}, \mathrm{r}=0$ and $\mathrm{R}_{0}$ as a parameter. Due to the reasons given previously in case of $\mathrm{w}_{\mathrm{o}}=10^{-4} \mathrm{~m}$ the maximum of the temperature is shifted towards greater $t$ values than $t=\Delta t_{p} / 4$. The increase of the temperature with increasing $\mathrm{R}_{\mathrm{o}}$ values is due to the explanation given in Fig. (3). The slow reduction of the temperature for $\mathrm{R}_{0}>5 \times 10^{-4} \mathrm{~m}$ is due to the radial temperature distribution. The appearance of a maximum followed by a minimum and then followed by a maximum for $\mathrm{R}_{0}=$ $\infty \mathrm{m}$ is due to the reasons given in Fig. (3). Due to the great width of the laser radiation more of the wings will be truncated leading to smaller temperature for $\mathrm{R}_{\mathrm{o}}=10^{-4} \mathrm{~m}$ than the linear case of $\mathrm{w}_{\mathrm{o}}=10^{-4} \mathrm{~m}$ part $[\mathrm{I}]$. For $\mathrm{R}_{0}=5 \times 10^{-4} \mathrm{~m}$ the increase of the width of the laser radiation reduces the intensity more than the gained from the increased amplification leading to smaller temperature than the linear case of $\mathrm{w}_{0}=10^{-4} \mathrm{~m}$. For the other $\mathrm{R}_{\mathrm{o}}$ values the increase of the width, due to the behavior of $\left(\beta_{L}-\alpha_{L}\right)$, leads to a greater temperature than the linear case. That the temperature in this case is greater than the case of $\mathrm{w}_{0}=10^{-4} \mathrm{~m}$ is due to the smaller width of the laser radiation after the minimum width compared with $\mathrm{w}_{\mathrm{o}}=10^{-4} \mathrm{~m}$ and the location of the minimum width is in this case closer to $\mathrm{z}=\mathrm{L}$.

Figure (8) represents the axial temperature distribution calculated at $t=\Delta t_{p} / 4, \mathrm{r}=0$ and $\mathrm{R}_{\mathrm{o}}$ as a parameter. For $\mathrm{R}_{\mathrm{o}}=10^{-4} \mathrm{~m}$ the temperature at $\mathrm{z}=0$ attains a greater value than its neighbors this value continue to decrease till the vicinity of the minimum width of the laser radiation where the temperature increases rapidly to reach its maximum value at $\mathrm{z}=0.06 \mathrm{~m}$. This behavior is explained in Fig. (4). After reaching the temperature its maximum value it begins to decrease. This is because of the increased width leading in one hand to the reduction of the intensity of the laser radiation and on the other hand, due to the negative value of $\left(\beta_{L}-\alpha_{L}\right)$, to cut off its wings. For the other $\mathrm{R}_{\mathrm{o}}$ values, the temperature at $\mathrm{z}=0$ is smaller than at greater $\mathrm{z}$ values. This is due to the behavior of $\left(\beta_{L}-\alpha_{L}\right)$ and the effect of self-focusing which leads the temperature for $\mathrm{R}_{0}=5 \times 10^{-4} \mathrm{~m}$ to have a maximum at $\mathrm{z}=0.07 \mathrm{~m}$. For the other $\mathrm{R}_{\mathrm{o}}$ values the maximum is at $\mathrm{z}=\mathrm{L}$. This is due to the great radial amplification which overcompensates the reduction of the intensity resulting from the increase of the radius as well as accumulation of the heat energy at small times (linear behavior). 

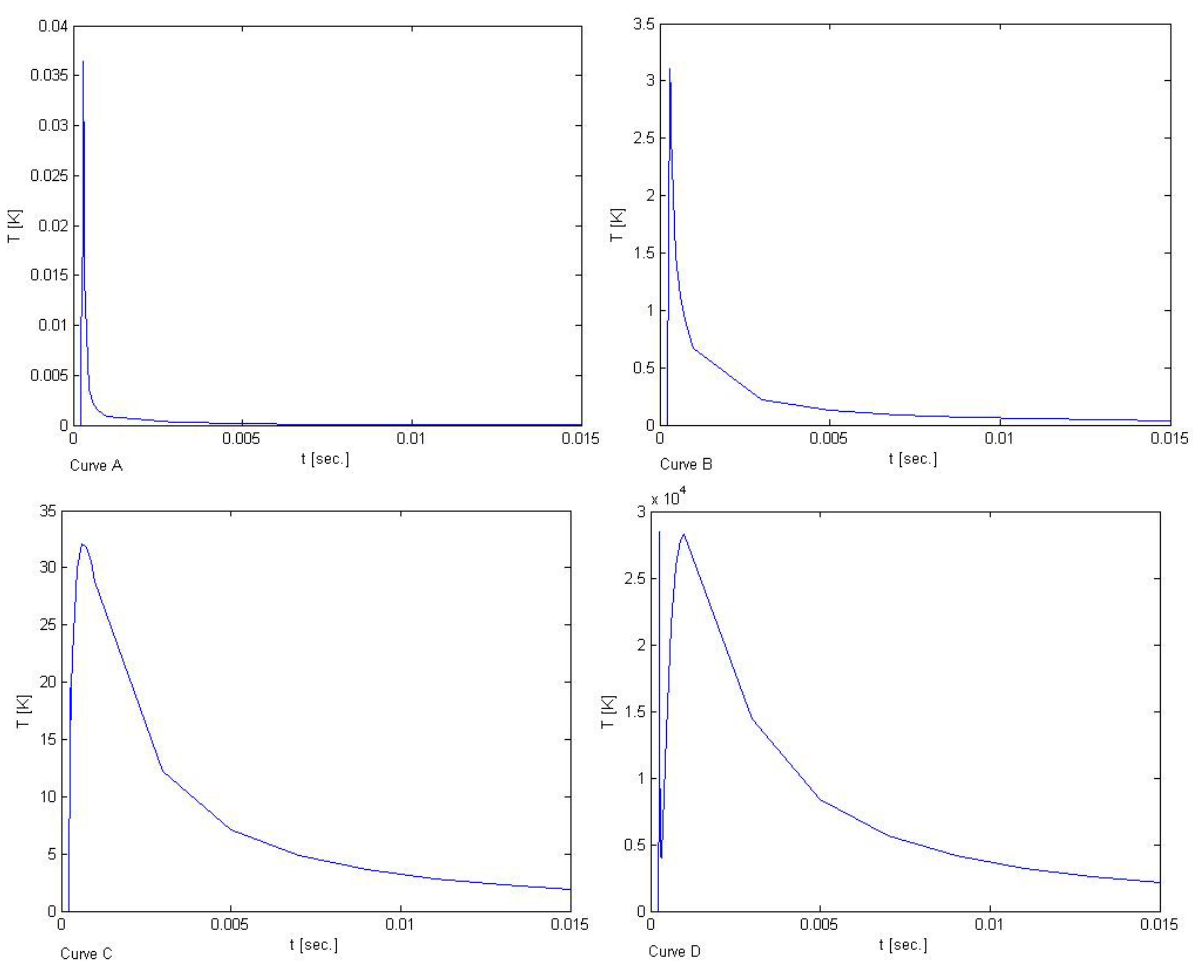

Fig. (7): The temporal temperature distribution induced by a laser beam having spatial intensity distribution of increasing width with increasing $\mathrm{z}$ - value (Gaussian beam) originating from a laser oscillator $0.1 \mathrm{~m}$ long, laying $0.1 \mathrm{~m}$ a part from a laser amplifier. The Gaussian beam has a width of $1.77 \times 10^{-4} \mathrm{~m}$ at the input of the laser amplifier. The calculation was carried out considering self-focusing at $\mathrm{z}=\mathrm{L}, \mathrm{r}=0$ and $\mathrm{R}_{0}$ as a parameter. Considering amplified power.

$\mathrm{R}_{0}=10^{-4} \mathrm{~m}$ curve $A \quad \mathrm{R}_{0}=5 \times 10^{-4} \mathrm{~m}$ curve $\mathrm{B} \quad \mathrm{R}_{0}=10^{-3} \mathrm{~m}$ curve $\mathrm{C}$

$\mathrm{R}_{0}=\infty \mathrm{m} \quad$ curve $\mathrm{D}$

Figure (9) represents the radial temperature distribution calculated at $t=\Delta t_{p} / 4, \mathrm{z}=\mathrm{L}$ and $\mathrm{R}_{0}$ as a parameter. The curves behave in relative distribution generally as those given in Fig. (5) except that the temperature in this case is greater. This is due to the greater cross-section of the laser beam allover the time except at times during which self-focusing occurs and at at which laser cross-sections are practically the same. Due to the smaller width of the laser radiation at $\mathrm{z}=\mathrm{L}$ and the greater distance over which the width of the laser radiation in this case is smaller than the case of self-focused Gaussian distribution having $w=10^{-4} \mathrm{~m}$ and represented in Fig. (5), the temperature in this case is higher. 

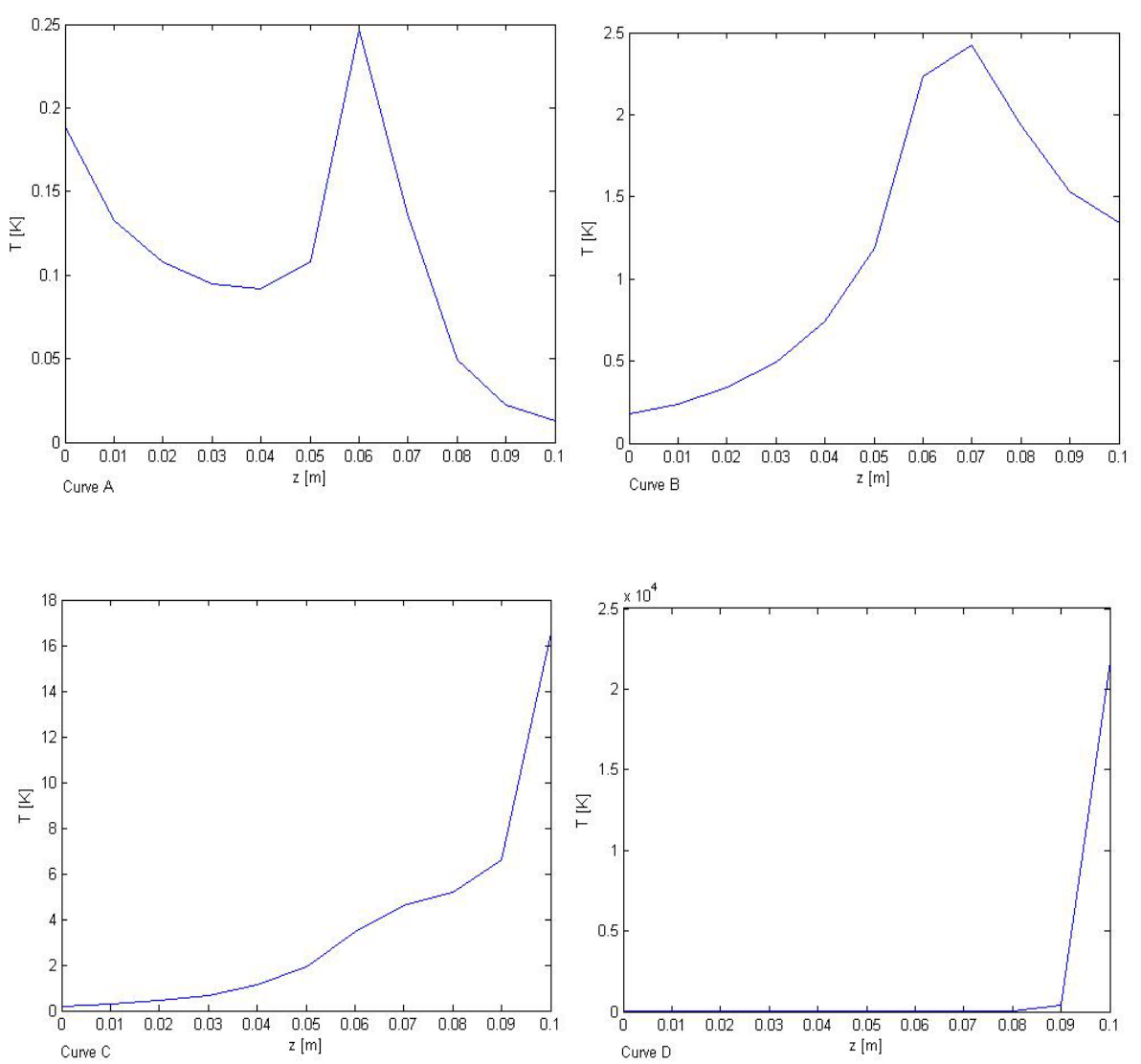

Fig. (8): The temperature distribution as a function of $\mathrm{z}$ induced by a laser beam having spatial intensity distribution of increasing width with increasing $\mathrm{z}$ - value (Gaussian beam) originating from a laser oscillator $0.1 \mathrm{~m}$ long, laying $0.1 \mathrm{~m}$ a part from a laser amplifier. The Gaussian beam has a width of $1.77 \times 10^{-4} \mathrm{~m}$ at the input of the laser amplifier. The calculation was carried out considering selffocusing at $t=\Delta t_{p} / 4, r=0$ and $R_{0}$ as a parameter. Considering amplified power. $\mathrm{R}_{0}=10^{-4} \mathrm{~m}$ curve $A \quad \mathrm{R}_{0}=5 \times 10^{-4} \mathrm{~m}$ curve $\mathrm{B} \quad \mathrm{R}_{0}=10^{-3} \mathrm{~m}$ curve $\mathrm{C}$ $\mathrm{R}_{0}=\infty \mathrm{m}$ curve $\mathrm{D}$ 

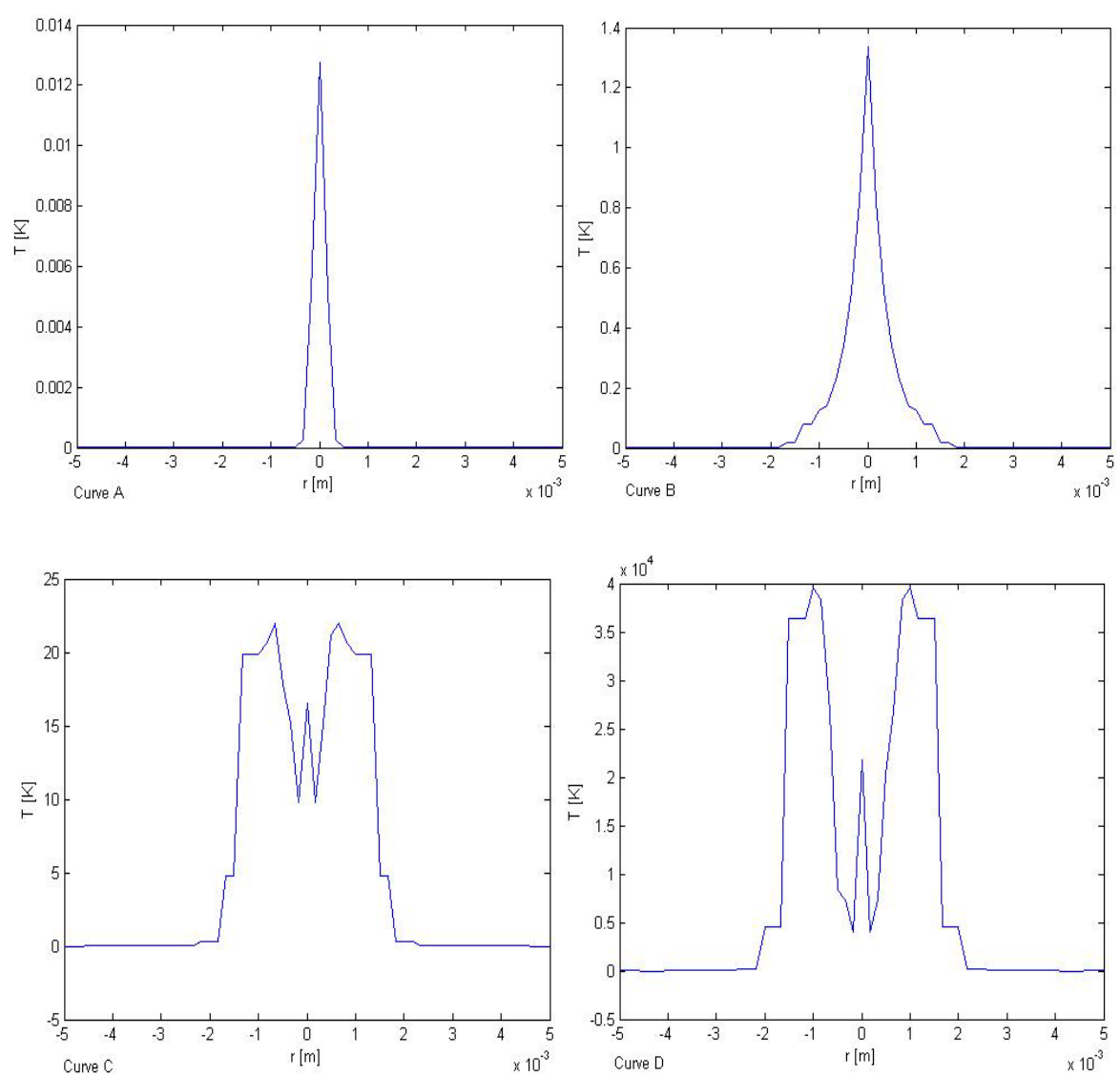

Fig. (9): The radial temperature distribution induced by a laser beam having spatial intensity distribution of increasing width with increasing $\mathrm{z}$ - value (Gaussian beam) originating from a laser oscillator $0.1 \mathrm{~m}$ long, laying $0.1 \mathrm{~m}$ a part from a laser amplifier. The Gaussian beam has a width of $1.77 \times 10^{-4} \mathrm{~m}$ at the input of the laser amplifier. The calculation was carried out considering selffocusing at $\mathrm{t}=\Delta \mathrm{t}_{\mathrm{p}} / 4, \mathrm{z}=\mathrm{L}$ and $\mathrm{R}_{\mathrm{o}}$ as a parameter. Considering amplified power.

$\mathrm{R}_{0}=10^{-4} \mathrm{~m} \quad$ curve $A \quad R_{0}=5 \times 10^{-4} \mathrm{~m} \quad$ curve $B_{0}=10^{-3} \mathrm{~m} \quad$ curve $\mathrm{C}$ $\mathrm{R}_{0}=\infty \mathrm{m}$ curve D 


\section{Conclusion:}

The location at which the minimum width was observed was found to move towards greater $\mathrm{z}$ values as the width of the laser radiation was increased. The axial temperature was found to increase with increasing $\mathrm{w}_{\mathrm{o}}$ value for $\mathrm{R}_{\mathrm{o}}>$ $5 \times 10^{-4} \mathrm{~m}$.

\section{Reference:}

1. Haber, F.E.and Cohen, M.I. ، ‘Solid-State Electtron" 11, (1968) 1176.

2. Ready, J. F. ،Effects of high power laser radiation " Acadimic press New York (1971) .

3. Breinan, E. M., Kear, B. H. and Banas, C. M. Processing materials with laser. Phys. Today 29 (1967) 44.

4. Charaschan, S. S., “Laser Industry" Van Nostrand, New York (1972) chaps. 4 and 5.

5. Porteus, J. D. and Decker, D. L., “ Metal mirror damage and precision diamond-turning". In laser induced damage in optical material (A. J. glass and A. H. Guenther, Eds) Academic press, New York (1980).

6. Porteus, J. D., Choyke, W. J. and Hollman, R. A., “ Pulsed laser damage characteristic of vapor-deposited copper mirrors on silicon carbide substrate". Appl. Opt. vol. 19, 451 (1980).

7. Koechner W. Solid-state laser engineering, 4th ed. Berlin: Springer, p. 381 (1992).

8. Comasky B, Moran BD, Albrecht GF and Beach RJ. Characterization of heat loading of Nd: doped YAG, YOS, YLF, and GGG excited at diode pumping wavelength. IEEE J Quantum Electron; 31, 1261 (1995).

9. Sliney JG, Leung KM, Birnbaum $M$ and Tucker AW. Lifetimes of the 4F3=2 state in Nd:YVO4. J. Appl Phys 50(5):3778-9 (1979).

10. P:stner $C$, Weber R, Weber HP, Merrazzi $S$ and Gruber R. Thermal beam distortion ins in end pumped Nd:YAG, Nd:GSGG and Nd:YLF rods. IEEE J Quantum Electron; 30, 1605 (1994).

11. Jabczynski JK, Kopczynski $K$ and Szczesniak A. Thermal lensing and thermal aberration investigations in diode-pumped lasers. Opt Eng; 35(12):3572 (1996).

12. V. Spagnolo, M.Troccoli, G. Scamarcio, C. Becker, G. Glastre and C. Sirtori " Facet temperature mapping of GaAs/AlGaAs quantum cascade lasers by photoluminescence microprobe " Optical Materils 17, 219 (2001).

13. G. Romo, T. Smy, D. Walkey and B. Reid "Modeling facet heating in ridge lasers " Microelectronics Reliability 43, 99 (2003). 
14. Kosterin, Andrey; Erwin, J. Kevin and Fallahi, Mahmoud; Mansuripur, Masud " Heat and temperature distribution in a claddingpumped, Er: Yb co-doped phosphate fiber " Review of Scientific Instruments, Volume 75, Issue 12, pp. 5166-5172 (2004).

15. Ready . G.F. "Effects due to absorption of laser radiation" J.Appl. Phys 36, 462 (1965).

16. Hassan. A. F. etal "Heating effects induced by a pulsed laser in a semiinfinite target in view of the theory of linear system" Optics \& Laser Technology Vol. 28 No. 5, 337 (1996).

17. Peter W. Milonni and Joseph H. Eberly "Lasers" Rochester, New York, John Wily \& Sons (1988).

18. M. M. El-Nicklawy, A. F. Hassan, El. M. A. Nasr, A.A. Hemida and S. M. El-Genedy "Two dimensional temperature distribution resulting from propagation of a light beam through an amplifying medium Part 1: linear case” To Be Published 